\title{
ANÁLISE DE ESPÍCULAS DE ESPONJAS COMO INDICADORES PALEOAMBIENTAIS EM SEDIMENTOS LACUSTRES NO OESTE DA BAHIA
}

\author{
GISELE BARBOSA DOS SANTOS \\ Curso de Geografia, Centro das Humanidades, UFOB, Rua Professor José Seabra de Lemos, 316, \\ Recanto dos Pássaros, 47808-021, Barreiras, BA, Brasil.gisele.barbosa@ufob.edu.br \\ PAULO DE TARSO AMORIM CASTRO \\ Departamento de Geologia, Escola de Minas, UFOP, Morro do Cruzeiro, s/n, 35400-000, Ouro Preto, MG, Brasil. \\ ptacastro@gmail.com \\ MAURO PAROLIN \\ Universidade Estadual do Paraná, Unespar, Av. Comendador Norberto Marcondes, 733, 87303-100, \\ Campo Mourão, PR, Brasil.mauroparolin@gmail.com
}

\section{LOYANA DOCIO}

Curso de Biologia, Departamento de Ciências Humanas, Campus IX, UNEB, BR-242, km 04, s/n, Flamengo, 47802-682, Barreiras, BA, Brasil.loydocio@gmail.com

\section{DIANDRA HOFFMANN COSTA}

Curso de Geografia, Centro das Humanidades, UFOB, Rua Professor José Seabra de Lemos, 316, Recanto dos Pássaros, 47808-021, Barreiras, BA, Brasil.diandrageo_hoffmann@hotmail.com

\begin{abstract}
ANALYSIS OF SPONGE SPICULES AS PALEOENVIRONMENTAL INDICATORS IN WESTERN BAHIA LACUSTRINE SEDIMENTS. The use of sponge spicules in sediments as proxy data has been successful in paleoenvironmental reconstructions. We recovered two sedimentary cores in two ponds located in San Francisco lowland in Western Bahia to understand the paleoclimatic and the lacustrine system dynamics. Sedimentary facies and granulometric analysis were perfomed. Also, organic matter content and presence of diatoms frustules were studied, besides the identification and quantification of sponge spicules detected in sediments, which were dated by ${ }^{14} \mathrm{C}$. The sediment age ranged between 8410 to $3929 \mathrm{yrs}$ cal BP, corresponding to the Holocene. Sponge spicules of the following species were found: Dosilia pydanieli, Heterorotula fistula, Metania spinata, Oncoslera sp., Radiospongilla inesi and Tubella variabilis. Analysis of the results suggest that the two lakes have a standardized climate pattern alternating between drier and wetter climates in relation to the current climate condition. However, the two lakes differ regarding the sedimentation rate and the microfossil content the pond of the northern portion showed lentic and lotic alternated phases, indicating a paleodrainage at the beginning of the upper Holocene, while the evidences in the southern pond of the lake system there are only lentic phases. Such evidences suggest that other physiographic factors and climate influenced the dynamics of these lakes, such as structure, litholog and tectonics of the ponds substrates.
\end{abstract}

Key words: Quaternary, Holocene, San Francisco lowland, sponges.

RESUMO - A utilização de espículas de esponjas em sedimentos como dado proxy vem obtendo sucesso em proposições de reconstruções paleoambientais. Para compreender as condições paleoclimáticas e dinâmicas do sistema lacustre, localizado na Depressão do São Francisco, no oeste do estado da Bahia, foram recuperados dois testemunhos em duas lagoas. Foram realizadas análises de fácies, ensaios granulométricos, de conteúdo de matéria orgânica e presença de diatomáceas, além da identificação e quantificação de espículas de esponjas detectadas nos sedimentos holocênicos, que foram datados por ${ }^{14} \mathrm{C}$. A idade das amostras dos sedimentos variou entre 8.410 a 3.929 cal. AP. Foram encontradas espículas das esponjas Dosilia pydanieli, Heterorotula fistula, Metania spinata, Oncoslera sp, Radiospongilla inesi e Tubella variabilis. As análises sugerem que as duas lagoas tiveram situações climáticas com alternância entre fases mais secas e mais úmidas em relação ao clima atual. No entanto, as duas lagoas diferem no que tange à taxa de sedimentação e conteúdo microfossilífero. Para a lagoa da porção norte os resultados evidenciaram alternância de fases lênticas e lóticas, indicando uma paleodrenagem no início do Holoceno superior, enquanto as evidências da lagoa ao sul do sistema lacustre indicam apenas fases lênticas. Tais evidências sugerem que outros fatores fisiográficos, além do clima, influenciaram a dinâmica dessas lagoas, como características estruturais, tectônicas e litológicas do substrato das lagoas.

Palavras-chave: Quaternário, Holoceno, Depressão do São Francisco, lagoas. 


\section{INTRODUÇÃO}

Espículas de esponjas vêm sendo utilizadas com sucesso como base para reconstrução paleoambiental do Quaternário em áreas tropicais úmidas, isso porque possuem a sílica biogênica em sua composição, o que as torna mais resistentes ao intemperismo químico, mesma característica dos fitólitos e frústulas de diatomáceas (Stevaux, 1994). A análise destes elementos pode ser considerada vantajosa quando comparada aos palinomorfos, tradicionalmente mais utilizados em pesquisas paleoambientais, já que a matéria orgânica é mais susceptível aos processos de oxidação e remobilização (Parolin et al., 2008). Além desta vantagem, estes autores salientam que por sua composição silicosa as espículas de esponjas podem ser preservadas em sedimentos mais grossos, como a areia.

Estas informações balizaram a escolha de espículas de esponjas e frústulas de diatomáceas como dados proxies neste trabalho, visto que as lagoas investigadas possuem caráter sazonal, com grande oscilação do nível de água, ou mesmo completa evaporação no período de estiagem, o que favorece a oxidação da matéria orgânica presente nos sedimentos. Neste sentido, Clarke (2003) destaca que além da importância paleoambiental, a opala biogênica é um componente importante na interação com o regolito, no que se refere aos processos de ciclagem da sílica, cimentação e processos de silificação, salientando que tem havido pouca integração de dados paleoambientais, paleontológicos, sedimentológicos e de ciência do solo relacionados a estudos do regolito.

No Brasil alguns trabalhos têm sido produzidos sobre a taxonomia de espículas de esponja fósseis em sedimentos de ambiente de águas continentais, com ênfase nos estudos paleoambientais e paleoecológicos em escalas locais, como salientam Kalinovski et al. (2016). A região Centro-Sul se destaca em número de pesquisas, principalmente nos Estados do Mato Grosso do Sul, Paraná e São Paulo, seguida da região norte, com estudos concentrados no Estado do Pará e apenas um trabalho na Região Nordeste, no Estado do Maranhão. Alguns estudos sobre espículas de esponjas e processos pedogenéticos são encontrados em Costa et al. (1992) em gleissolos húmicos no Estado de Minas Gerais e Lani et al. (2007) em gleissolos tiomórficos no Estado do Espírito Santo.

Diante deste panorama, a partir de uma abordagem multidisciplinar, este trabalho tem por objetivo compreender a evolução da paisagem lacustre na região do médio Rio São Francisco, tendo por base: (i) as datações absolutas $\left({ }^{14} \mathrm{C}\right)$; (ii) os registros sedimentológicos e micropaleontológicos (representados pelas espículas de esponja), capazes de gerar indícios potenciais sobre o quadro cronológico e fases paleoambientais. Aliado a este fato há uma escassez de estudos sobre depósitos quaternários na Depressão do São Francisco, mesmo esta unidade perfazendo uma área superior a 800.000 hectares no oeste do Estado da Bahia. Não há relatos de análise de espículas de esponja em sedimentos lacustres na Bahia anteriormente a esta pesquisa, como pode ser observado no levantamento realizado por Kalinovski et al. (2016). Portanto, este é o primeiro trabalho realizado com o uso de espécies de esponjas de água doce presentes neste estado durante o Holoceno.

\section{ÁREA DE ESTUDO}

O sistema lacustre investigado situa-se nos municípios de Santa Rita de Cássia e no Distrito de Taguá pertencente a Cotegipe, na porção oeste do Estado da Bahia (Figura 1). Nessa região são encontradas mais de 2.000 lagoas com diferentes morfologias e tamanhos (Santos \& Castro, 2016). Geotectonicamente, essa área está situada no noroeste do Cráton do São Francisco, uma importante unidade geotectônica formada desde o Arqueano, que adquiriu estabilidade ao final do Evento Transamazônico (Alkmim, 2004). O Cráton do São Francisco é margeado por faixas móveis (Almeida, 1981), que são regiões envolvidas na tectogênese brasiliana do final do Neoproterozoico. A Faixa Móvel do Rio Preto é o limite norte da área de estudo.

Geomorfologicamente o sistema lacustre localiza-se na Depressão do São Francisco, cuja conformação é de uma área plana com pequena amplitude altimétrica, que varia de $400 \mathrm{e}$ $510 \mathrm{~m}$, circundada pelo Chapadão do Urucuia e pela Serra do Espinhaço Setentrional, denominada localmente de Serra do Boqueirão. A Depressão do São Francisco se desenvolve ao longo da drenagem do Rio São Francisco, onde predominam formas de relevo aplainadas, assentando-se nela sedimentos neogênicos e quaternários que têm como substrato as rochas metapelíticas e carbonáticas do Grupo Bambuí (Egydio-Silva, 1987). O Chapadão do Urucuia é a feição mais conspícua da região ocorrendo ao redor da Depressão do São Francisco, com exceção de seu limite leste representado pela Serra do Boqueirão (Espinhaço Setentrional). Nestas duas unidades, o Chapadão do Urucuia e a Serra do Boqueirão, encontram-se as nascentes dos principais canais fluviais que drenam a região, o Rio Grande e o Rio Preto (Figura 1). Com exceção destes dois rios, toda a rede de drenagem que flui na área do sistema lacustre possui comportamento intermitente.

O clima da região é do tipo Aw (Clima Tropical ou Tropical de Savana) pela classificação de Köppen, que, de acordo com Alvares et al. (2014), é marcadamente sazonal, com verão chuvoso, que se estende de outubro a abril e inverno seco, de maio a setembro, com temperaturas médias que variam entre $20^{\circ} \mathrm{C}$ e $26^{\circ} \mathrm{C}$ (Gaspar, 2006). Os índices pluviométricos anuais oscilam entre 800 e $1100 \mathrm{~mm}$, configurando-se um clima subúmido seco de acordo com Moreira \& Silva (2010). A porção onde está inserido o sistema lacustre é uma área de transição entre os biomas do cerrado e da caatinga denominada “Área de Tensão Ecológica" (IBGE,1992).

\section{MATERIAL E MÉTODOS}

Após a abertura dos dois testemunhos de sondagem, o material foi dividido e coletado amostras em intervalos de 4 $\mathrm{cm}$, colocadas para secar ao ar. As fácies sedimentares em cada testemunho foram individualizadas baseando-se na textura do material, cor, arranjo, organização, espessura, presença e tipo de estruturas sedimentares primárias, presença e tipos de concreções, mosqueamentos e matéria orgânica.

Para estabelecer a cronologia dos testemunhos analisados foram enviadas três amostras para datações pelo método do 

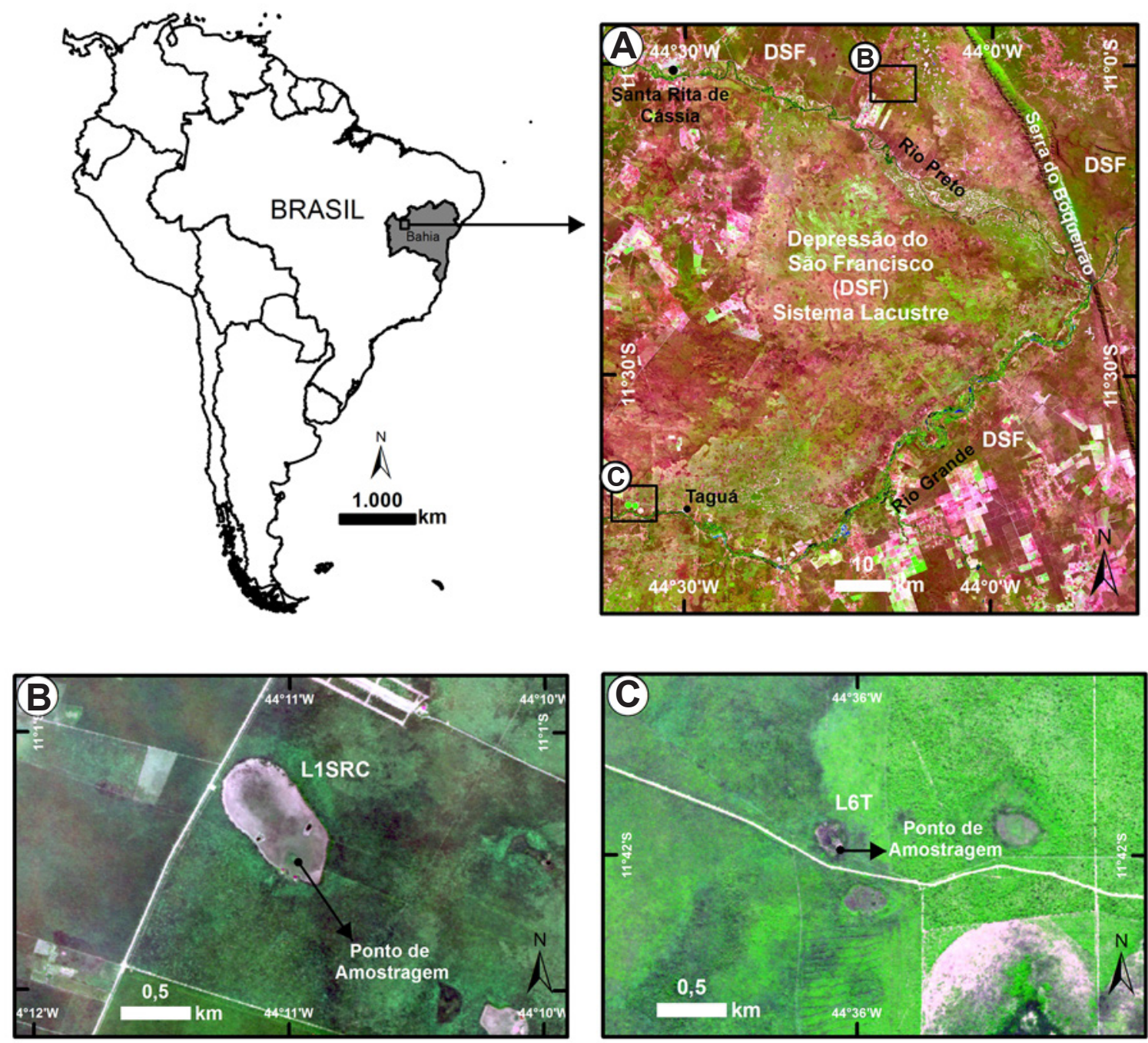

Figura 1. Localização da área de estudos. A, lagoas na Depressão do São Francisco (Landsat 8 OLI30 m - RGB654 - 05/2015); B-C, detalhamento dos pontos de coleta dos testemunhos (Rapideye - $5 \mathrm{~m}$ - 10/2010).

Figure 1. Location of the study area. A, lakes at the São Francisco lowlands (Landsat 8 OLI30 m - RGB654 - 05/2015); B-C, detailing of the collection points of the sediment cores (Rapideye - $5 \mathrm{~m}$ - 10/2010).

Carbono 14 (University of Georgia/Center for Applied Isotope Studies - Georgia/EUA), e posteriormente calibrados (Calib $7 ®)$. Os sedimentos datados foram coletados na profundidade de $24 \mathrm{~cm}$ para o testemunho L6T e nas profundidades de 32 e $64 \mathrm{~cm}$ no testemunho L1SRC.

Para atestar a presença e abundância de espículas foram coletadas amostras de sedimentos em duas lagoas (L1SRC e L6T). A lagoa com sufixo SRC se localiza na Bacia do Rio Preto no município de Santa Rita de Cássia; já a lagoa com o sufixo T se localiza na Bacia do Rio Grande, no distrito de Taguá, pertencente ao município de Cotegipe. A coleta foi realizada por meio de sondagem realizada por percussão com auxílio de marreta, utilizando-se tubos de PVC (diâmetro de $50 \mathrm{~mm}$ ) e alumínio (diâmetro de $90 \mathrm{~mm}$ ). Foram recuperados dois testemunhos, um na lagoa L1SRC de $96 \mathrm{~cm}$ e outro na lagoa L6T de $36 \mathrm{~cm}$.

O percentual de matéria orgânica foi obtido pelo método da calcinação "Loss of Ignition", adaptado de Davies
(1974). Este método consiste na pesagem de $10 \mathrm{~g}$ de cada amostra, que é levada à mufla e aquecida por $5 \mathrm{~h}$ a $550^{\circ} \mathrm{C}$. Posteriormente, a amostra é pesada e a diferença entre o peso inicial $(10 \mathrm{~g})$ e final corresponde ao teor de matéria orgânica. As rotinas para recuperação de espículas de esponjas foram realizadas no Laboratório de Estudos Paleoambientais da Fecilcam (Lepafe) da Universidade Estadual do Paraná (UNESPAR - Campus de Campo Mourão, PR), seguindo a metodologia descrita por Volkmer-Ribeiro \& Turcq (1996). Para a confecção de lâminas foram destorroadas amostras e separados $2 \mathrm{~g}$, dispostos em tubos de ensaio e em seguida fervidos com ácido nítrico $65 \%\left(\mathrm{HNO}_{3}\right)$. Posteriormente o sedimento foi lavado com água destilada por meio de quatro centrifugações. Para a montagem das lâminas de microscopia, o sedimento foi centrifugado mais uma vez em álcool $70 \%$. $\mathrm{O}$ resíduo resultante foi adicionado com pipeta mecânica (25 $\mu$ l) sobre lâminas que, após a secagem, foram cobertas com entelan ${ }^{\circledR}$ e lamínula. No total foram preparadas 300 
lâminas, que estão arquivadas no Laboratório de Estudos Paleoambientais da UNESPAR com os códigos L.36;C.3 e L.37;C.3. As lâminas foram analisadas e registradas no microscópio óptico ZEISS Scope A1 ${ }^{\circledR}$ (câmera fotográfica acoplada-AxioCamMRc5). Os registros de elementos endoesqueletais constituídos por sílica das espículas de esponjas presentes foram avaliados conforme as categorias descritas por Volkmer-Ribeiro \& Pauls (2000), sendo: (i) megascleras, espículas que integram as bases da rede esqueletal e geralmente são as maiores espículas presentes e na maioria das vezes, apesar de serem mais abundantes nas amostras, não permitem distinções específicas nítidas, podendo, em alguns casos, diferenciar alguns gêneros; (ii) microscleras, espículas de tamanho reduzido que possuem valor taxonômico na caracterização de gêneros e de algumas espécies; (iii) gemoscleras, espículas que recobrem as gêmulas das esponjas continentais e que constituem o caráter necessário para a caracterização específica. A identificação das espécies foi operada com base na coleção de esponjas do Lepafe, além de literatura especializada (e.g. Volkmer-Ribeiro \& Parolin, 2010; Volkmer-Ribeiro \& Pauls, 2000; Pinheiro et al., 2003; Pinheiro, 2007; Pinheiro \& Nicacio, 2012). A contagem destes elementos foi feita por três transectos por lâminas em três lâminas para cada profundidade avaliada.

Embora as espículas de esponjas sejam o foco principal, as frústulas de diatomáceas também foram contadas, visto que nas lâminas utilizadas para a identificação das espículas estes elementos proxies estavam visíveis e foram então utilizados para atestarem a presença de água no ambiente. No entanto, a identificação das diatomáceas foi operada somente ao nível de grupo, tendo por base os trabalhos de Leandrini et al. (2010) Souza et al. (2007), entre outros.

\section{RESULTADOS E DISCUSSÃO}

\section{Datação $\left({ }^{14} \mathrm{C}\right)$}

Os sedimentos datados na lagoa L1SRC correspondem ao Holoceno médio a superior, $4.738 \mathrm{cal}$. AP $(64 \mathrm{~cm}$ de profundidade) e $3.929 \mathrm{cal}$. AP (36 cm de profundidade). Os sedimentos da lagoa L6T correspondem ao Holoceno inferior, $8.410 \mathrm{cal}$. AP ( $24 \mathrm{~cm}$ de profundidade).

\section{Granulometria}

No testemunho da lagoa L1SRC, até $36 \mathrm{~cm}$ de profundidade houve predomínio de areia $(39,9 \%)$ com presença de seixos arredondados de quartzo de $2 \mathrm{~cm}$. Destaca-se que até esta profundidade foi registrado o maior percentual de silte $(26,9 \%)$ e o menor percentual de argila $(33,3 \%)$ dentre todos os testemunhos. De $36 \mathrm{~cm}$ de profundidade até $60 \mathrm{~cm}$ houve um decréscimo na porcentagem de areia e silte $(14,5$ e $14,1 \%$ respectivamente) e uma elevação no percentual de argila (71,4\%). De $60 \mathrm{~cm}$ até $96 \mathrm{~cm}$ houve pequeno acréscimo de areia e silte (19,8 e 15,5\% respectivamente) e pequeno decréscimo de argila, mas com porcentagem ainda alta $(64,6 \%)$.

No testemunho da lagoa L6T, de $36 \mathrm{~cm}$ até a profundidade de $24 \mathrm{~cm}$ houve predominância de argila (77,5\%), seguidos de $12,5 \%$ de areia e menor porcentagem de silte $(9,9 \%)$. Este padrão perdura nos $12 \mathrm{~cm}$ até a base do testemunho, ocorrendo aumento na porcentagem de areia $(22,9 \%)$ e decréscimo nas porcentagens de silte e argila $(8,6$ e $68,5 \%)$, respectivamente (Figuras 2, 3).

\section{Matéria orgânica}

No testemunho da lagoa L1SRC os percentuais de matéria orgânica variaram de 4,2 a $11,5 \%$, com os mais altos valores na parte central (entre 32 a $44 \mathrm{~cm}$ ), 10,5 a 11,5\%. A partir desta profundidade os percentuais caíram até a base do perfil. No testemunho da lagoa $L 6 T$ os valores foram de 7,2 a 11,7\% com um aumento na direção do topo. Destaca-se a presença de valores mais significativos próximos ao topo do perfil (de 4 a $8 \mathrm{~cm}$ ), com 11,7 e 10,2\% (Figuras 2,3).

\section{Análise das fácies}

A Figura 2 ilustra três fácies identificadas no testemunho da lagoa L1SRC: fácies 1, presente na parte inferior do testemunho, apresentou menor teor de matéria orgânica $(7,4 \%)$, coloração cinza-claro, estrutura maciça muito argilosa e ausência de raízes. A fácies 2, de depósito intermediário, apresenta coloração cinza-claro, estrutura maciça muito argilosa, possuindo a maior porcentagem desta granulação em relação às outras fácies $(71,4 \%)$, mosqueamentos raros e um acréscimo de matéria orgânica $(8,6 \%)$ em relação à fácies anterior, com rara presença de raízes. A fácies 3, topo da sequência, apresentou cor marrom-cinza-clara, mosqueamento mais abundante que a fácies anterior, e um pequeno decréscimo no teor de matéria orgânica $(8,2 \%)$; nesta fácies foram registrados abundantes seixos de quartzo arredondados de aproximadamente $2 \mathrm{~cm}$ e presença abundante de raízes. Esta fácies apresenta estrutura maciça com granulação franco-argilosa. As duas fácies mais antigas desta lagoa possuem características de ambiente lêntico e a fácies mais recente de ambiente lótico, principalmente pelo aumento do tamanho das partículas depositadas.

Os sedimentos recuperados da lagoa L6T (Figura 3) apresentaram duas fácies: A fácies 1, na base do testemunho, com coloração marrom-clara-acinzentada e presença de precipitação dendrítica de óxido de manganês de cores pretas e raros mosqueamentos. A fácies 2, mais recente, com coloração vermelho-amarelada, mosqueamento mais abundante em relação à anterior e presença de raízes oxidadas. Os sedimentos coletados em todo o testemunho possuem textura muito argilosa, o que indica baixa energia no ambiente deposicional. A porcentagem de argila aumenta significativamente da fácies 1 para a fácies 2 de $68,5 \%$ para $77,5 \%$.

\section{Espículas de esponja}

Os microfósseis de esponjas identificados incluem: Dosilia pydanieli Volkmer-Ribeiro, 1992; Heterorotula fistula Volkmer-Ribeiro \& Motta, 1995; Metania spinata Carter, 1881a; Oncosclera Volkmer-Ribeiro, 1970; Radiospongilla inesi Nicacio \& Pinheiro, 2011; Tubella variabilis Carter, $1881 \mathrm{~b}$. 


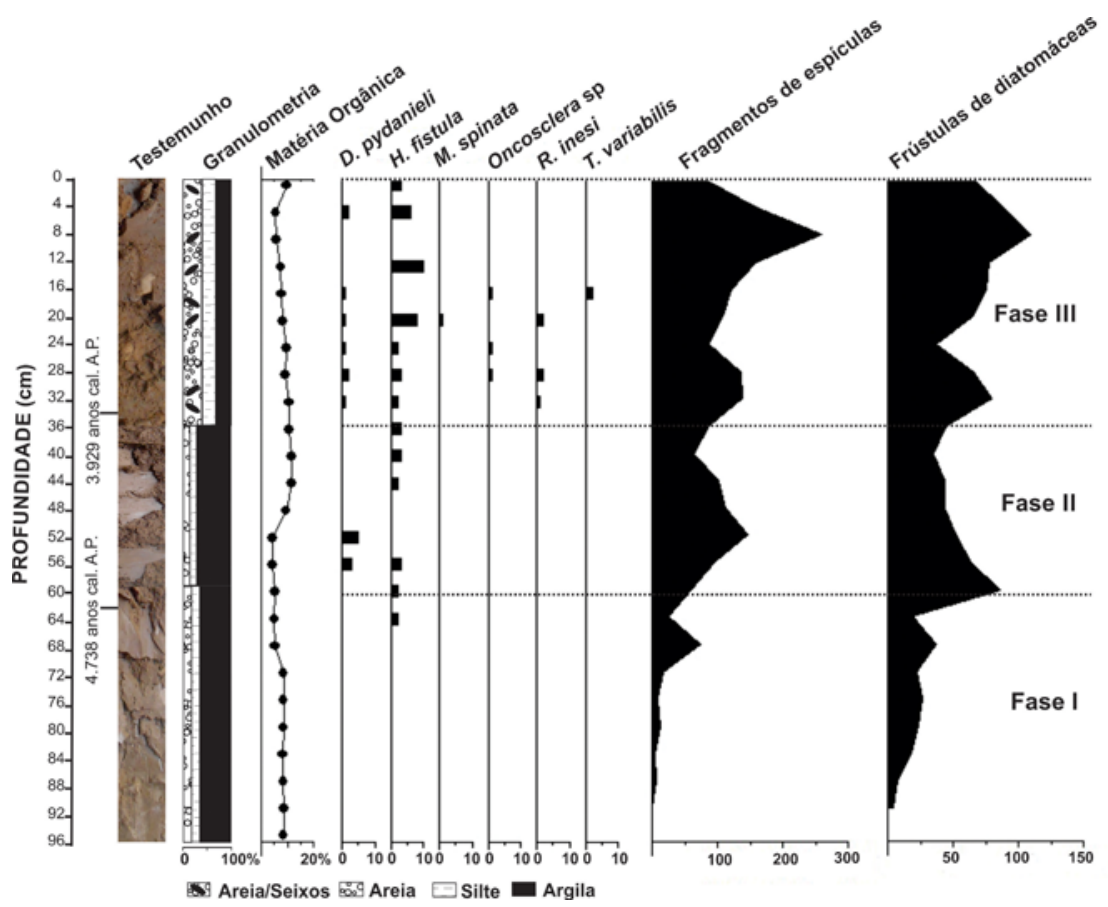

Figura 2.Testemunho L1SRC: datações, granulometria, percentuais de matéria orgânica, ocorrência de espículas de esponjas, fragmentos de espículas e frústulas de diatomácea.

Figure 2. Sediment core L1SRC: dating, granulometry, organic matter percentage, presence of sponge spicules, spicules fragments and diatoms frustules.

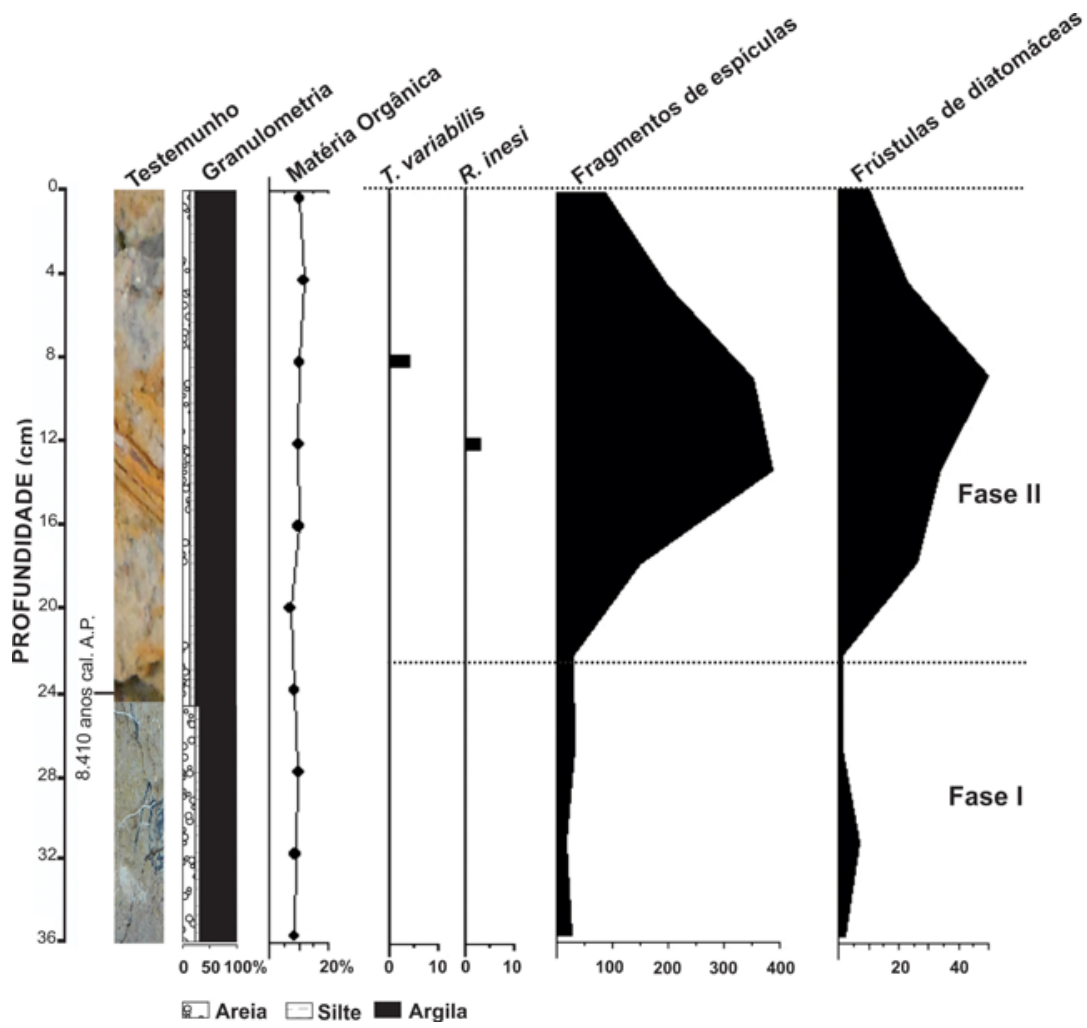

Figura 3.Testemunho L6T: datações, granulometria, percentuais de matéria orgânica, ocorrência de espículas de esponjas, fragmentos de espículas e frústulas de diatomácea.

Figure 3. Sediment core L6T: dating, granulometry, organic matter percentage, presence of sponge spicules, spicules fragments and diatoms frustules. 
Este trabalho adota a nomenclatura do gênero Tubella como sinonímia do Trochospongilla, seguindo a proposta de Pinheiro \& Nicacio (2012), onde Trochospongilla passou a ser sinônimo júnior de Tubella. Os diferentes tipos e morfologias das espículas são apresentados na Figura 4 e as distribuições e quantificação das espécies de esponjas nos sedimentos das lagoas estão representadas graficamente nas Figuras 2, 3.

Espículas de Dosilia pydanieli, Heterorotula fistula, Metania spinata, Tubella variabilis e Radiospongilla inesi indicam condições típicas de ambiente lêntico (VolkmerRibeiro et al., 1998; Parolin, 2006; Nicacio et al., 2011; Kuerten et al., 2013).

As características gerais do ambiente de ocorrência de Dosilia pydanieli foram descritas por Volkmer-Ribeiro (1999) como lagos rasos com presença abundante de macrófitas, as quais incrustam raízes e galhos e outros materiais submersos, protegidas da luz solar. Parolin (2006) atesta que esta foi a única encontrada nas lagoas na margem ocidental do Rio Paraná e no Estado do Mato Grosso do Sul. A presença de Heterorotula fistula foi atestada por Parolin (2006) no sedimento de lagoas no Terraço Taquarussu, margem direita do Rio Paraná e por Kuerten et al. (2013) em lagoas na planície do Rio Nabileque no sul do Pantanal, nos dois locais analisados as características descritas indicaram ambiente lêntico. Almeida et al., (2009), ao estudarem lagoas cársticas no noroeste do Estado de Minas Gerais, constatam que a colonização por $H$. fistula foi favorável em lagoas com condições de coluna de água bastante reduzida, onde os processos de dissolução cárstica foram mais lentos, formando uma bacia mais rasa.
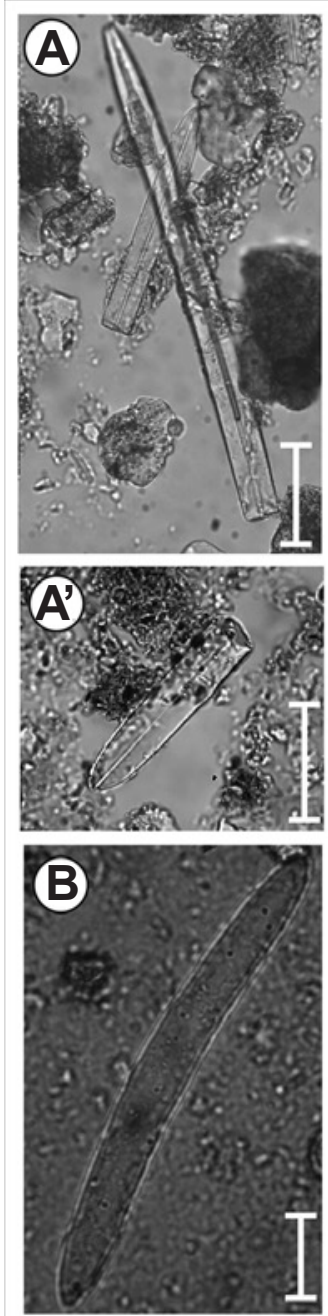
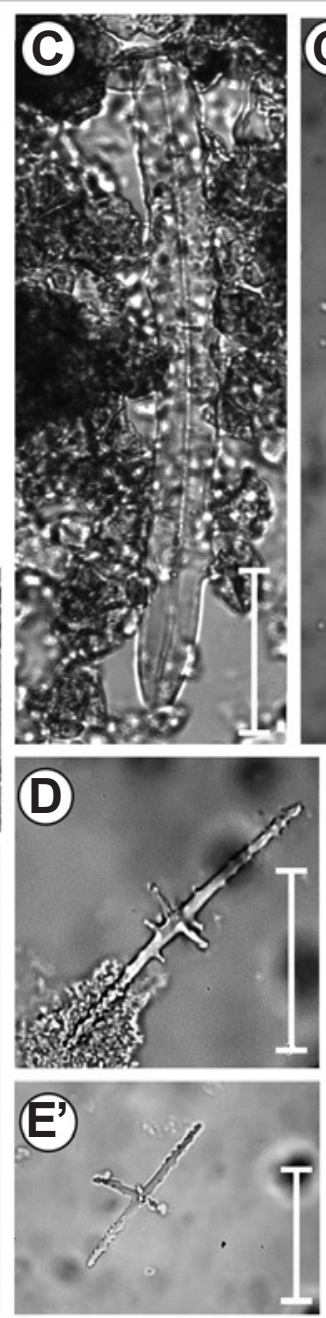
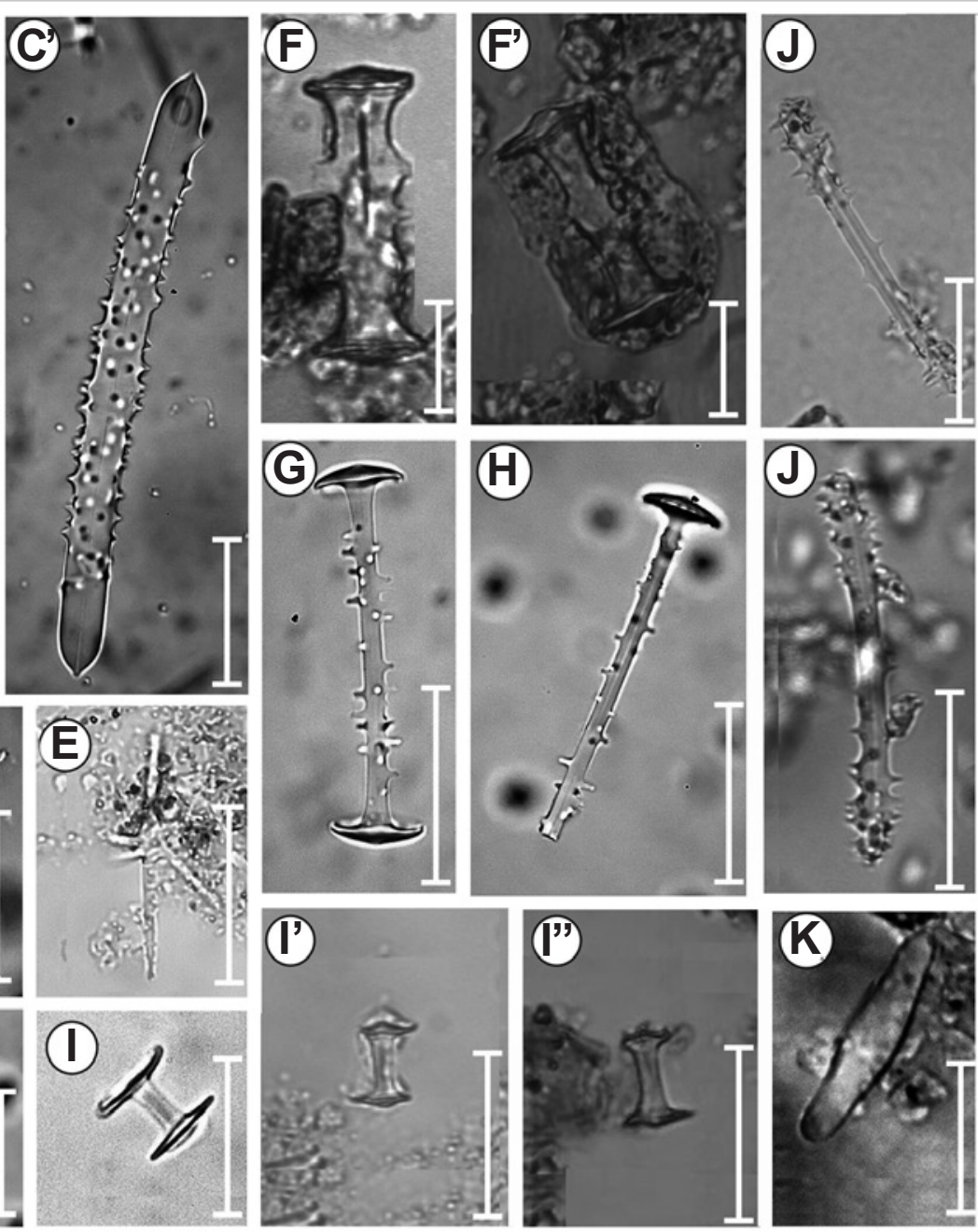

Figura 4. Fotomicrografias de espículas de esponjas continentais: A-A', fragmentos de megascleras; B, megasclera; C-C', megasclera beta de Metania spinata; D, microsclera de Metania spinata; E-E', microsclera de Dosilia pydanieli; F-F', gemosclera de Heterorotula fistula; G, gemosclera de Dosilia pydanieli; $\mathbf{H}$, fragmento de gemosclera de Dosilia pydanieli; I-I", gemosclera de Tubella variabilis; J, gemosclera de Radiospongilla inesi; K, gemosclera de Oncosclera sp. Escalas $=20 \mu \mathrm{m}$.

Figure 4. Photomicrographs of continental sponge spicules: A-A', fragment of megascleres; B, megasclere; C-C', megasclere beta of Metania spinata; D, microsclere of Metania spinata; E-E', microsclere of Dosilia pydanieli; F-F', gemmosclere of Heterorotula fistula; G, gemmosclere of Dosilia pydanieli; H, fragment of gemmosclere of Dosilia pydanieli; I-I”, gemmosclere of Tubella variabilis; J, gemmosclere of Radiospongilla inesi; K, gemmosclere of Oncosclera sp. Scale bars $=20 \mu \mathrm{m}$. 
O ambiente de adaptação de Metania spinata foi descrito por Volkmer-Ribeiro \& Motta (1995) como pequenas lagoas, incrustada em macrófitas, folhas, galhos e troncos submersos, que podem possuir água levemente acidificada. Sua ocorrência foi amplamente registrada no Bioma Cerrado, na América do Sul (Parolin et al., 2010). Tubella variabilis também está relacionada a ambientes lênticos, sendo bioindicadora de locais de baixa luminosidade devido à abundância de macrófitas (Volkmer-Ribeiro et al., 2007). Os ambientes descritos para a ocorrência de Radiospongilla inesi são lênticos e semi-lóticos em águas rasas, tendo sido registradas em lagoas e riachos no Estado de Pernambuco por Nicacio et al. (2011).

A presença de espículas do gênero Oncoslera é indicativo de ambientes lóticos ou semi-lóticos em que as esponjas se aderem a substratos rochosos (Volkmer-Ribeiro \& Pauls, 2000). Diferentes espécies deste gênero foram descritas em vários canais fluviais no Brasil, como nos rios Negro e Orinoco (Volkmer-Ribeiro \& Almeida, 2005) e nos rios Araguaia e Paraná (Batista et al., 2007). Estes últimos autores também registraram espículas deste gênero em ambiente lêntico no Lago do Goiaba no Estado do Mato Grosso.

\section{Interpretação paleoambiental}

Os resultados obtidos por meio dos sedimentos das duas lagoas do sistema lacustre da Região Oeste da Bahia permitem afirmar que a evolução geomorfológica para cada um deles difere tanto no que diz respeito à cronologia, quanto à dinâmica sedimentar de seus depósitos. Os registros em L1SRC permitiram uma análise mais detalhada, já que a taxa média de sedimentação inferida foi de $11 \mathrm{~cm}$ a cada mil anos, enquanto que para L6T esta taxa foi de $2,9 \mathrm{~cm}$ a cada mil anos.

Ford \& Williams (2007) ao estudarem depressões em áreas cársticas elucidam que tais feições, quando são formadas por dissolução, iniciam seu desenvolvimento a partir de pontos de infiltração e que a ampliação dessas depressões, tanto em planta quanto em rebaixamento, é resultante da conexão entre pequenos condutos abertos por dissolução ao longo de planos de acamamento e fraturas das rochas. Cruz \& Alkmim (2006) e Uhlein et al. (2011) admitem que a deformação das rochas na área de estudo durante o Ciclo Brasiliano foi mais intensa na porção norte, onde se localiza L1SRC e vai progressivamente diminuindo para a porção sul, onde se encontra L6T e é pequena a expressão de estruturas rúpteis, com falhas e fraturas. Esta diferença na intensidade de fraturamento pode ser a causa pela qual na porção norte as lagoas se desenvolvem mais, tanto em área quanto em profundidade em relação a porção sul. Assim, a geração de espaço pelo processo cárstico, que resulta na ampliação das depressões, favorece a taxas de sedimentação, que são maiores na região onde se localiza L1SRC.

Quanto ao conteúdo micropaleontológico, foram registrados nas duas lagoas, de microfósseis de cinco espécies e um gênero de esponjas, sendo todos os tipos registrados em L1SRC, enquanto que em L6T duas espécies foram encontradas. A maior abundância de espículas de esponjas nas lagoas pode ser correlacionada à textura dos sedimentos, pois a maior parte dos corpos silicosos biogênicos foram registrados nas porções silto-arenosas. Nas faixas de granulação menor, (fração argila), os processos geoquímicos são mais intensos, devido à presença de íons adsorvidos e com ligações fracas com argilominerais, favorecendo a dissolução das espículas, corroborando os trabalhos de Clarke (2003), Costa et al. (2010) e Martins (2012).

Além de maior porcentagem de silte nos depósitos em L1SRC a fração areia também se mostrou superior. Destaca-se a presença de seixos arredondados, denotando maior energia no transporte destes sedimentos, relacionados a canais fluviais (Miall, 1985). Diante das diferenças encontradas nas duas lagoas a descrição de suas fases evolutivas será apresentada separadamente.

\section{L1SRC}

O intervalo do testemunho entre 96 e $64 \mathrm{~cm}$ de profundidade, referido como Fase I, representa pouco tempo de residência de água a tal ponto que não permitiu a colonização por esponjas. A presença de frústulas de diatomáceas sugere uma condição mais lêntica do que lótica. O tempo de residência de água aumenta a partir de 4.738 cal. anos AP, no entanto sofre variação significativa até o topo da Fase II, como alternância de duas espécies de espículas e aumento de frústulas de diatomáceas no início desta fase e declínio no final, onde uma amostra foi datada por ${ }^{14} \mathrm{C}(4.738$ cal. anos AP), apresentando uma boa compatibilidade com as condições climáticas atestadas por De Oliveira et al. (1999) em pesquisas palinológicas no médio São Francisco, a leste da área de estudo, indicando condições semiáridas entre 6.790-6.230 anos AP.

A presença de espículas de Dosilia pydanieli e Heterorotula fistula sugere que a área constituiu uma lagoa sazonal típica, com oscilações do nível de água indicativo da ocorrência de secas bem definidas. Na Fase III destaca-se a presença de espículas de Oncosclera e $R$. inesi normalmente associadas a ambientes lóticos ou semi-lóticos, atestando o aumento da umidade. A presença destas espécies conjugada aos dados de granulometria desta fase denota ambiente lótico.

Pode-se perceber que quando se finaliza a ocorrência de Oncosclera e $R$. inesi, tem-se em concomitância, o único registro de espículas de T. variabilis. Esta esponja é típica de lagoas sombreadas, ou seja, cercada por vegetação mais densa (Machado et al., 2012), o que sugere condições mais úmidas em relação às condições climáticas atuais. No início desta fase marcada pela profundidade de $\sim 34 \mathrm{~cm}$ a datação obtida (3.929 anos cal. AP) corrobora os estudos de De Oliveira et al. (1999), que apontam condições mais úmidas por volta de 4.535 anos AP.

\section{L6T}

O intervalo do testemunho entre 36 e $24 \mathrm{~cm}$ de profundidade, referido como Fase I, representa pouquíssimo tempo de residência de água, não permitindo a colonização por esponjas e indicando ambiente com pouca umidade. A presença de frústulas de diatomáceas sugere condição lêntica, com lagoas rasas. No final desta fase, $(24 \mathrm{~cm})$ a datação $(8.410$ anos cal. AP) e as condições ambientais apontadas acima estão 
em consonância com os estudos de De Oliveira et al. (1999) em estudos palinológicos tanto a leste da área de estudo (Rio São Francisco), que registraram declínio da umidade por volta de 8.910 anos AP, quanto similares na região do Brasil Central, como os registros de Salgado-Labouriau et al. (1997) de clima seco entre 11.500-6.500 anos AP e Barbieri (2001), que aponta, clima mais seco e instalações de estações secas mais prolongadas por volta de 7.900 anos AP. Por fim, estudos palinológicos de Behling (2002) em sedimentos da Lagoa da Confusão no Estado do Tocantins apontam que o clima estava marcadamente mais seco (baixa precipitação e longa estação seca anual) durante o período de 14.000 e 5.460 anos AP. A base da Fase II é marcada por um estágio mais úmido atestado pelo aumento de frústulas de diatomáceas e fragmentos de espículas de esponjas, o que denota maior tempo de residência de água. A cerca de $14 \mathrm{~cm}$ de profundidade observa-se um pico de fragmentos de espículas e frústulas, bem como a ocorrência de espículas de R. inesi e T. variabilis. Em vista disso, pode-se inferir que a taxa de sedimentação nesta lagoa foi constante e para esta profundidade estima-se uma idade aproximada de 4.800 anos AP, coincidindo com o registro de aumento de umidade também apontado em L1SRC e corroborados por De Oliveira et al. (1999).

A ocorrência de espículas de esponjas inteiras tem sido interpretada como prova da sua origem autigênica, ou seja, as esponjas habitaram o local ou suas proximidades onde seus microfósseis foram encontrados. Por outro lado, o registro de espículas fragmentadas vem sendo associado à origem alogênica, ou seja, material transportado a longas distâncias de sua área fonte, principalmente em consequência do atrito por conta de transporte eólico relacionados a períodos secos (Wilding \& Drees et al., 1968). Estes mesmos autores acrescentam que os fragmentos de espículas podem ser resultantes de misturas nos depósitos por biopedoturbação ou por rachaduras nos solos. Estas rachaduras, por sua vez, podem ser interpretadas pelo caráter expansivo de argilas em diferentes condições de umidade, capaz de fragmentar as espículas (Frisone et al., 2014). No caso das duas lagoas estudadas, é mais provável que tal processo esteja ligado à compactação das argilas nos depósitos, visto que o número de fragmentos aumentou em ambas nas fases interpretadas como condições climáticas mais úmidas e com fácies mais argilosas. É possível notar que o número de fragmentos de espículas em L6T é praticamente o dobro em relação a L1SRC, o mesmo ocorre, para a porcentagem de argila, visto que L1SRC possui fácies relacionada a estrato maciço com seixos dispersos em matriz fina típica de ambiente fluvial e L6T apresenta fácies com lama constituída por acamamento maciço típico de ambiente lacustre (Miall, 1985).

\section{CONCLUSÕES}

A utilização de espículas de esponjas de água doce como dados proxy permitiu a interpretação paleoambiental, com ênfase nos efeitos climáticos e hídricos ligados à evolução geomorfológica do sistema lacustre na região do médio São Francisco, no oeste do Estado da Bahia, no período holocênico. As fases nas quais as espículas de esponja estavam ausentes indicaram pouca residência de água no ambiente, relacionada a climas mais secos, em torno de 8.410 anos cal. AP e fase lacustre apresentando maior diversificação de espécies de esponjas em 4.738 anos cal. AP. Uma fase de influência fluvial ocorreu por volta de 3.929 anos cal. AP na lagoa situada ao norte do sistema lacustre. A utilização das frústulas de diatomáceas como dados proxies complementares quantitativos, auxiliaram na interpretação sobre a presença de água nas lagoas; destarte futuras pesquisas qualitativas podem enriquecer as interpretações paleoambientais desta região. As amostras estudadas nos testemunhos obtidos em duas lagoas revelaram que o fator climático foi semelhante para as duas áreas do sistema lacustre. Isso permitiu a distinção de outros fatores de influência para as diferentes taxas de sedimentação e conteúdo microfossilífero entre elas, podendo-se atribuir a influência do padrão estrutural e litológico do arcabouço geológico em que essas duas lagoas se formaram, bem como às características texturais distintas de seus depósitos.

\section{AGRADECIMENTOS}

Os autores agradecem ao Laboratório de Estudos Paleoambientais da FECILCAM/UNESPAR, Campo Mourão, e a CAPES mediante concessão de bolsa durante os dois primeiros anos de Doutorado do primeiro autor, bem como a concessão de bolsa de estágio sênior ao terceiro autor (processo CAPES nº 0471-16-0).

\section{REFERÊNCIAS}

Alkmim, F.F. 2004. O que faz de um cráton um cráton? O Cráton do São Francisco e as revelações almeidianas ao delimitá-lo. In: V. Mantesso-Neto; A. Bartorelli; C.D.R. Carneiro \& B.B BritoNeves (eds.) Geologia do continente sul-americano: evolução da obra de Fernando Flávio Marques de Almeida, Beca, p. 17-35.

Almeida, A.C.S.; Volmer-Ribeiro, C.; Varajão A.F.D.C.; Gomes, N.S. \& Varajão, C.A.C. 2009. Espículas de esponjas continentais nos sedimentos cenozoicos do noroeste de Minas Gerais, como indicadores paleoambientais. Revista Brasileira de Paleontologia, 12:123-138. doi:10.4072/rbp.2009.2.03

Almeida, F.F.M. 1981. O Cráton do Paramirim e suas relações com o do São Francisco. In: SIMPÓSIO SOBRE O CRÁTON DO SÃO FRANCISCO E SUAS FAIXAS MARGINAIS, 1, 1981. Anais, Salvador, p. 1-10.

Alvares, C.L.; Stape, J.L.; Sentelhas, P.C.; Gonçalves, J.L.M. \& Sparovek, G. 2014. Köppen's climate classification map for Brazil. Meteorologische Zeitschrift, 22:711-728. doi:10.1127/0941-2948/2013/0507

Barberi, M. 2001. Mudanças paleoambientais na região dos cerrados do Planalto Central durante o Quaternário tardio: o estudo da lagoa Bonita, DF. Programa de Pós-Graduação em Geologia Sedimentar, Universidade de São Paulo, Tese de doutorado, 210 p.

Batista, T.C.A.; Volkmer-Ribeiro, C. \& Melão, M.G.G. 2007. Espongofauna da Área de Proteção Ambiental Meandros do Rio Araguaia (GO, MT, TO), Brasil, com descrição de Heteromeyenia cristalina sp. nov. (Porifera, Demospongiae). Revista Brasileira de Zoologia, 24:608-630. doi:10.1590/ S0101-81752007000300013 
Behling, H. 2002. Late Quaternary vegetation and climate dynamics in southeastern Amazonia inferred from lagoa da Confusão in Tocantins State, northern Brazil. Amazoniana, 17:27-39.

Carter, H.J. 1881a. History and classification of the known species of Spongilla. Annals and Magazine of Natural History series, 5:77-107.

Carter, H.J. 1881b. History and classification of the known species of Spongilla. Annals and Magazine of Natural History series, 7:247-250.

Clarke, J. 2003. The occurrence and significance of biogenic opal in the regolith. Earth-Science Reviews, 60:175-194. doi:10.1016/ S0012-8252(02)00092-2

Costa, L.M.; Keller, W.D. \& Johns, W.D. 1992. Espículas de esponja em solos de João Pinheiro, Minas Gerais. Revista Ceres, 39:597-603.

Costa, L.M.; Santos, R.F.; Schafer, C.E.G.R.; Moreau, A.M.S.S. \& Moreau, M.S. 2010. Ocorrência de corpos silicosos em horizontes superficiais de solos de diferentes ecossistemas. Revista Brasileira de Ciência do Solo, 34:871-879. doi:10.1590/ S0100-06832010000300028

Cruz, S.C.P. \& Alkmim, F.F. 2006. The tectonic interaction between the Paramirim Aulacogen and the Araçuaí belt, São Francisco craton region, Eastern Brazil. Anais da Academia Brasileira de Ciências, 78:151-173. doi:10.1590/S0001-37652006000100014

Davies, B.E. 1974. Loss-on-ignition as an estimate of soil organic matter. Soil Science Society of America Journal, 38:347-353. doi:10.2136/sssaj1974.03615995003800010046x

De Oliveira, P.E; Barreto, A.M.F. \& Suguio, K. 1999. Late Pleistocene/Holocene climatic and vegetational history of the Brazilian Caatinga: the fossil dunes of the middle São Francisco River. Palaeogeography, Palaeoclimatology, Palaeoecology, 152:319-337. doi:10.1016/S0031-0182(99)00061-9

Egydio-Silva, M.E. 1987. O sistema de dobramentos Rio Preto e suas relações com o cráton do São Francisco. Instituto de Geociências, Universidade de São Paulo, Tese de doutorado, 141 p.

Ford, D.C. \& Williams, P.W. 2007. Karst Hydrology and geomorphology. Chichester, John Wiley and Sons Ltd, 562 p.

Frisone, V.; Pisera, A.; Hajdu, E.; Preto, N.; Zorzi, F. \& Zorzin, R. 2014. Isolated spicules of Demospongiae from Mt. Duello (Eocene, Lessini Mts., northern Italy): preservation, taxonomy, and depositional environment. Facies, 60:883-904. doi:10.1007/ s10347-014-0407-3

Gaspar, M.T.P. 2006. Sistema Aqüifero Urucuia: caracterização regional e propostas de gestão. Instituto de Geociências, Universidade de Brasília, Tese de doutorado, 204 p.

IBGE. 1992. Instituto Brasileiro de Geografia e Estatística. Manual Técnico da Vegetação Brasileira. Rio de Janeiro, IBGE, 92 p.

Kalinovski, E.C.Z.; Parolin, M. \& Souza-Filho E.E. 2016. Estágio das pesquisas sobre esponjas continentais na América do Sul: enfoque para a produção científica no Brasil. Revista Terrae Didática, 12:4-18.

Kuerten, S.; Parolin, M.; Assine, M.L. \& Mcglue, M.M. 2013. Sponge spicules indicate Holocene environmental changes on the Nabileque River floodplain, southern Pantanal, Brazil. Journal of Paleolimnology, 49:171-183. doi:10.1007/s10933-012-9652-z

Lani, J.L.; Schwandes, L.; Rezende, S.B.; Lani, J.A.; Amaral, E.F.; Cunha, A.M. \& Costa, L.M. 2007. Uso de opalas biogênicas na identificação de sedimentos lacustres e marinhos. Revista Brasileira de Ciência do Solo, 31:827-832. doi:10.1590/S010006832007000400023

Leandrini, J.A.; Cavalcanti, E.V. \& Rodrigues, L. 2010. Diatomáceas. In: M. Parolin; C. Volkmer-Ribeiro \& J.A. Leandrini (eds.)
Abordagem ambiental interdisciplinar em bacias hidrográficas no estado do Paraná, Editora da Fecilcam, p. 131-158.

Machado, V.S.; Volkmer-Ribeiro, C. \& Iannuzzi, R. 2012. Inventary of the Sponge Fauna of the cemitério Paleolake, Catalão, Goiás, Brazil. Anais da Academia Brasileira de Ciências, 84:17-34. doi/:10.1590/S0001-37652012000100004

Martins, C.M. 2012. Gênese, formas de carbono e sílica biogênica de solos sob formações estacionais do semiárido de Minas Gerais e Bahia. Departamento de Solos, Universidade Federal de Viçosa, Tese de doutorado, 115 p.

Miall, A.D. 1985. Architectural-element analysis: a new method of facies analysis applied to fluvial deposits. Earth-Science Reviews, 22:261-308. doi:10.1016/0012-8252(85)90001-7

Moreira, M.C. \& Silva, D.D. 2010. Atlas hidrológico da Bacia hidrográfica do Rio Grande. Barreiras, Gazeta Santa Cruz, 80 p.

Parolin, M. 2006. Paleoambientes e paleoclimas no final do Pleistoceno e no Holoceno no Sudeste do Estado do Mato Grosso do Sul. Programa de Pós-Graduação em Ecologia de Ambientes Aquáticos Continentais, Universidade Estadual de Maringá, tese de doutorado, $120 \mathrm{p}$.

Parolin, M.; Volkmer-Ribeiro, C. \& Leandrini J.A. 2010. Abordagem ambiental interdisciplinar em bacias hidrográficas no Estado do Paraná. $1^{\mathrm{a}}$ ed. Campo Mourão, Editora da Fecilcam, 158 p.

Parolin, M.; Volkmer-Ribeiro, C. \& Stevaux, J.C. 2008. Use of spongofacies as a proxy for river-lake paleohydrology in Quaternary deposits of central-western Brazil. Revista Brasileira de Paleontologia, 11:187-198. doi:10.4072/rbp.2008.3.05

Pinheiro, U.S. 2007. Contribuições à taxonomia e biogeografia das esponjas de águas continentais brasileiras. Museu Nacional, Universidade Federal do Rio de Janeiro, Tese de doutorado, $220 \mathrm{p}$.

Pinheiro, U.S.; Hajdu, E. \& Caballero, M.E. 2003. Três novos registros de esponjas (Porifera, Demospongiae): para águas continentais do Estado de São Paulo. Boletim do Museu Nacional, Nova Série, Zoologia, 498:1-14.

Pinheiro, U.S. \& Nicacio, G. 2012. Resurrection and redefinition of the Genus Tubella (Porifera: Spongillidae) with a worldwide list of valid species. Zootaxa, 3269:65-68.

Salgado-Labouriau, M.L.; Casseti, V.; Ferraz-Vicentini, K.R.; Martin, L.; Soubiès, F.; Suguio, K. \& Turcq, B. 1997. Late Quaternary vegetational and climatic changes in cerrado and palm swamp from Central Brazil. Palaeogeography, Palaeoclimatology, Palaeoecology, 128:215-226. doi:10.1016/ S0031-0182(96)00018-1

Santos, G.B. \& Castro, P.T.A. 2016. Sedimentological characteristics and geochemistry of lake waters of basin, west of Bahia State (Brazil). International Journal of River Basin Management, 14:431-440. doi:10.1080/15715124.2016.1215323

Souza, G.S.; Koening, M.L.; Leça, E.E. \& Coêlho, M.P.C.A. 2007. Diatomáceas indicadoras de paleoambientes do Quaternário de Dois Irmãos, Recife, PE, Brasil. Acta Botanica Brasilica, 21:521-529. doi:10.1590/S0102-33062007000300002

Stevaux, J.C. 1994. Upper Paraná River (Brazil) geomorphology and paleoclimatology. Quaternary International, 21:143-161. doi:10.1016/1040-6182(94)90028-0

Uhlein, A.; Caxito, F.A.; Sanglard. J.C.D.; Uhlein, G. \& Suckau, G.L. 2011. Estratigrafia e tectônica das faixas neoproterozóicas da porção norte do Cráton do São Francisco. Geonomos, 19:8-31. doi:10.18285/geonomos.v19i1.57

Volkmer-Ribeiro, C. 1992. The freshwater sponges in some peatbog ponds in Brazil. Amazoniana, 12:317-335. 
Volkmer-Ribeiro, C. 1999. Esponjas. In: C.A. Joly \& C.E.M. Bicudo (eds.) Biodiversidade do Estado de São Paulo síntese do conhecimento ao final do século $X X$. Invertebrados de água doce, FAPESP, p. 1-19.

Volkmer-Ribeiro, C. \& Almeida, F.B. de. 2005. As esponjas do Lago Tupé. In: E. Santos-Silva; F.M. Aprile; V.V. Scudeller \& S. Melo (eds.) BioTupé: meio fisico, diversidade biológica e sociocultural do Baixo Rio Negro, Amazônia Central, INPA, p. 123-134.

Volkmer-Ribeiro, C.; De Rosa-Barbosa, R. \& Mostardeiro, C.C. 2007. Esponjas. In: F.G. Becker; R.A. Ramos, \& A. Moura (eds.) Biodiversidade da região dos Butiazais de Tapes e da Lagoa do Casamento, Planície Costeira do Rio Grande do Sul, MMA/SBF, p. 154-161.

Volkmer-Ribeiro, C. \& Maciel, S.B. 1983. New freshwater sponges from Amazonian waters. Amazoniana, 8:255-264.

Volkmer-Ribeiro, C. \& Motta, J.F.M. 1995. Esponjas formadoras de Espongilitos em lagoas no Triângulo Mineiro e adjacências, com indicação de preservação de habitat. Biociências, 3:145-169.

Volkmer-Ribeiro, C.; Motta J.F.M. \& Callegaro, V.L.M. 1998. Taxonomy and distribution of Brazilian spongillites. In: Y. Watanabe \& N. Fusetani (eds.) Sponge sciences-multidisciplinary perspectives, Springer, p. 271-278.
Volkmer-Ribeiro, C. \& Parolin, M. 2010. As esponjas. In: M. Parolin; C. Volkmer-Ribeiro \& J.A. Leandrini (eds.) Abordagem ambiental interdisciplinar em bacias hidrográficas no Estado do Paraná, Editora da Fecilcam, p. 105-130.

Volkmer-Ribeiro, C. \& Pauls, S.M. 2000. Esponjas de agua dulce (Porifera, Demospongiae) de Venezuela. Acta Biologica Venezuelica, 20:1-28.

Volkmer-Ribeiro, C. \& Turcq, B. 1996. SEM analysis of siliceous spicules of a fresh water sponge indicate paleoenvironmental changes. Acta Microscopica, 5:186-187.

Wilding, L.P. \& Drees, L.R. 1968. Distribution and implications of sponge spicules in surficial deposits in Ohio. Ohio Journal of Science, 68:92-99.

Received in June, 2016; accepted in November, 2016. 